\title{
Erratum to: Feasibility of Cognitively-Based Compassion Training (CBCT) for breast cancer survivors: a randomized, wait list controlled pilot study
}

\author{
Sally E. Dodds ${ }^{1}$ - Thaddeus W. W. Pace ${ }^{2}$ Melanie L. Bell ${ }^{3} \cdot$ Mallorie Fiero $^{3}$. \\ Lobsang Tenzin Negi ${ }^{4}$ Charles L. Raison ${ }^{1} \cdot$ Karen L. Weihs ${ }^{1}$
}

Published online: 8 September 2015

(C) Springer-Verlag Berlin Heidelberg 2015

Erratum to: Support Care Cancer (2015)

DOI: $10.1007 / \mathbf{s 0 0 5 2 0 - 0 1 5 - 2 8 8 8 - 1}$

Table 3. The words "Intervention - Control $(95 \% \mathrm{CI})$ in the top row refer to the headings in the row beneath labeled "Post" and "1-month FU" in the last two columns of the table.

The 8 th of 10 columns in this table refers to the 1-month FU for the control group.

Table 4. The label for the second column is "post-intervention" and the label for the third column is " 1 -month followup"

The online version of the original article can be found at http://dx.doi.org/ 10.1007/s00520-015-2888-1.

Sally E. Dodds

sdodds1@email.arizona.edu

1 University of Arizona College of Medicine, Tucson, AZ 85724, USA

2 University of Arizona College of Nursing, Tucson, AZ 85721, USA

3 University of Arizona Mel and Enid Zuckerman College of Public Health, Tucson, AZ 85724, USA

4 Emory University, Atlanta, GA 30322, USA 
Table 3 Means, standard deviations and differences between intervention and control for all psychosocial and biomarker outcomes at baseline, postintervention, and at 4-weeks FU

\begin{tabular}{|c|c|c|c|c|c|c|c|c|c|}
\hline \multirow[t]{3}{*}{ Outcome } & \multicolumn{7}{|c|}{ Mean (SD) } & \multicolumn{2}{|c|}{ Intervention - Control $(95 \% \mathrm{CI})$} \\
\hline & \multicolumn{4}{|c|}{ Intervention } & \multicolumn{3}{|l|}{ Control } & \multirow[b]{2}{*}{ Post } & \multirow[b]{2}{*}{ 1-month FU } \\
\hline & $\begin{array}{l}\text { Possible } \\
\text { range }\end{array}$ & $\begin{array}{l}\text { Baseline } \\
(N=12)\end{array}$ & $\begin{array}{l}\text { Post } \\
(N=12)\end{array}$ & $\begin{array}{l}1 \text {-mo FU } \\
(N=11)\end{array}$ & $\begin{array}{l}\text { Baseline } \\
(N=16)\end{array}$ & $\begin{array}{l}\text { Post } \\
(N=16)\end{array}$ & $\begin{array}{l}1 \text {-mo FU } \\
(N=11)\end{array}$ & & \\
\hline Perceived stress & $0-16$ & $5.4(2.1)$ & $3.5(1.5)$ & $3.5(2.7)$ & $5.4(2.9)$ & $4.7(2.5)$ & $5.1(3.0)$ & $-1.2(-2.5,0.2)$ & $-1.6(-3.1,-0.2)^{*}$ \\
\hline Depression & $0-30$ & $6.4(4.6)$ & $2.8(3.1)$ & $4.2(5.2)$ & $6.3(5.7)$ & $6.5(6.3)$ & $5.5(5.0)$ & $-3.7(-6.3,-1.1)^{* *}$ & $-1.3(-4.2,1.6)$ \\
\hline FCR: Triggers & $0-32$ & $14.3(6.3)$ & $12.4(5.6)$ & $14.2(8.2)$ & $13.3(5.4)$ & $14.6(6.9)$ & $12.5(5.8)$ & $-2.2(-6.0,1.6)$ & $1.7(-2.4,5.8)$ \\
\hline FCR: Severity & $0-36$ & $15.2(5.6)$ & $12.9(6.6)$ & $14.2(6.9)$ & $15.1(8.0)$ & $13.8(6.8)$ & $13.7(8.5)$ & $-0.9(-2.9,1.2)$ & $0.6(-1.7,2.8)$ \\
\hline FCR: Psychological distress & $0-16$ & $4.9(4.2)$ & $3.3(3.1)$ & $3.7(3.4)$ & $4.6(2.7)$ & $3.4(2.3)$ & $3.3(4.5)$ & $-0.1(-1.51 .3)$ & $0.4(-1.2,2.0)$ \\
\hline FCR: Functioning Impairments & $0-32$ & $2.7(3.5)$ & $1.2(1.9)$ & $3.0(3.2)$ & $2.6(3.7)$ & $2.5(3.0)$ & $1.7(2.7)$ & $-1.3(-2.5-0.1)^{*}$ & $1.3(-0.1,2.7)$ \\
\hline FCR: Insight & $0-12$ & $1.4(2.1)$ & $0.9(2.0)$ & $0.7(1.5)$ & $1.2(2.0)$ & $1.2(1.8)$ & $1.1(2.1)$ & $-0.3(-0.8,0.2)$ & $-0.3(-0.9,0.3)$ \\
\hline Traumatic stress: Intrusion & $0-4$ & $0.7(0.8)$ & $0.6(0.6)$ & $0.4(0.8)$ & $0.6(0.3)$ & $0.6(0.4)$ & $0.5(0.3)$ & $-0.1(-0.3,0.2)$ & $-0.1(-0.3,0.2)$ \\
\hline Traumatic stress: Avoidance & $0-4$ & $1.0(1.0)$ & $0.6(0.6)$ & $0.8(0.8)$ & $0.9(0.8)$ & $0.9(0.8)$ & $0.7(0.8)$ & $-0.3(-0.6,-0.02)^{*}$ & $0.1(-0.2,0.4)$ \\
\hline Traumatic stress: Hyper-arousal & $0-4$ & $0.6(0.6)$ & $0.3(0.4)$ & $0.4(0.7)$ & $0.5(0.5)$ & $0.4(0.5)$ & $0.4(0.4)$ & $-0.1(-0.3,0.2)$ & $-0.003(-0.3,0.3)$ \\
\hline Traumatic stress: Global & $0-16$ & $2.2(2.2)$ & $1.5(1.4)$ & $1.7(2.1)$ & $2.1(1.4)$ & $1.9(1.2)$ & $1.6(1.3)$ & $-0.4(-1.0,0.2)$ & $0.04(-0.6,0.7)$ \\
\hline Loneliness & $20-80$ & $38.3(10.6)$ & $34.5(9.4)$ & $35.5(10.2)$ & $38.8(16.2)$ & $37.4(15.4)$ & $37.9(16.6)$ & $-2.9(-7.7,2.0)$ & $-2.5(-7.9,3.0)$ \\
\hline Mindfulness & $10-40$ & $28.8(4.1)$ & $31.9(4.2)$ & $31.2(4.8)$ & $28.6(5.5)$ & $28.3(5.0)$ & $28.1(5.3)$ & $3.6(1.2,6.0)^{*}$ & $3.1(0.4,5.8)^{*}$ \\
\hline Gratitude & $6-42$ & $36.1(4.9)$ & $38.1(5.8)$ & $38.2(5.3)$ & $35.7(6.4)$ & $37.5(5.0)$ & $37.0(5.7)$ & $0.5(-1.9,3.0)$ & $1.2(-1.5,3.9)$ \\
\hline Vitality/fatigue & $0-100$ & $54.6(6.6)$ & $56.2(5.8)$ & $53.6(6.9)$ & $54.5(8.9)$ & $50.7(9.4)$ & $53.3(9.1)$ & $5.5(1.5,9.6)^{* *}$ & $0.3(-4.2,4.9)$ \\
\hline Bodily pain & $0-100$ & $48.2(10.2)$ & $51.6(8.1)$ & $50.1(10.3)$ & $48.4(9.8)$ & $49.6(10.2)$ & $52.0(7.0)$ & $2.0(-3.1,7.0)$ & $-1.9(-7.5,3.8)$ \\
\hline Physical well-being & $0-100$ & $50.4(7.0)$ & $50.9(7.6)$ & $49.7(7.6)$ & $50.6(9.8)$ & $51.1(8.8)$ & $54.0(4.9)$ & $-0.1(-3.2,2.9)$ & $-4.3(-7.7,-0.9)^{*}$ \\
\hline Mental well-being & $0-100$ & $49.6(5.9)$ & $50.4(5.4)$ & $50.9(7.1)$ & $48.9(11.7)$ & $48.4(10.6)$ & $46.5(10.4)$ & $2.0(-2.4,6.5)$ & $4.4(-0.6,9.3)$ \\
\hline \multicolumn{10}{|l|}{ Biomarkers } \\
\hline Salivary cortisol (AM) & & $-1.3(0.4)$ & $-1.1(0.3)$ & $-1.3(0.5)$ & $-1.3(0.3)$ & $-1.2(0.5)$ & $-1.2(0.5)$ & $0.1(-0.2,0.4)$ & $-0.01(-0.3,0.3)$ \\
\hline Salivary cortisol (PM) & & $-3.0(0.5)$ & $-3.3(0.9)$ & $-2.9(0.8)$ & $-3.0(0.8)$ & $-3.0(0.8)$ & $-2.8(1.0)$ & $-0.3(-0.9,0.3)$ & $-0.01(-0.6,0.6)$ \\
\hline Change in salivary cortisol $/ \mathrm{hr}^{\mathrm{a}}$ & & $-0.1(0.1)$ & $-0.2(0.1)$ & $-0.1(0.1)$ & $-0.1(0.1)$ & $-0.1(0.1)$ & $-0.1(0.1)$ & $-0.04(-0.1,0.005)$ & $-0.02(-0.1,0.04)$ \\
\hline
\end{tabular}

$S D$ standard deviation, $C I$ Confidence Interval, FCR Fear of Cancer Recurrence

${ }^{a}$ Salivary cortisol $(\mathrm{N})$ : Intervention $(12,12,12)$; Control $(15,13,14)$

$*<0.05$

$* *<0.01$ 
Table 4 Partial correlation of total practice time (in minutes) with psychosocial and biomarker outcomes at post-intervention and 4-weeks FU (accounting for baseline values) in the CBCT $\operatorname{Arm}(n=12)$

\begin{tabular}{|c|c|c|}
\hline Outcome & $\begin{array}{l}\text { Post intervention } \\
\text { Correlation }(95 \% \mathrm{CI})\end{array}$ & $\begin{array}{l}\text { One month follow-up } \\
\text { Correlation }(95 \% \mathrm{CI})\end{array}$ \\
\hline Perceived stress & $-0.27(-0.75,0.39)$ & $-0.01(-0.64,0.62)$ \\
\hline Depression & $0.17(-0.48,0.70)$ & $-0.26(-0.77,0.44)$ \\
\hline FCR: Triggers & $-0.16(-0.72,0.52)$ & $-0.14(-0.71,0.53)$ \\
\hline FCR: Severity & $-0.56(-0.87,0.07)$ & $-0.65(-0.91,-0.03)$ \\
\hline FCR: Psychological distress & $-0.33(-0.78,0.33)$ & $-0.65(-0.91,-0.04)$ \\
\hline FCR: Functioning impairments & $-0.13(-0.51,0.68)$ & $-0.20(-0.74,0.49)$ \\
\hline FCR: Insight & $-0.26(-0.74,0.41)$ & $-0.15(-0.71,0.53)$ \\
\hline Traumatic stress: Intrusion & $0.41(-0.53,0.66)$ & $0.05(-0.60,0.66)$ \\
\hline Traumatic stress: Avoidance & $0.10(-0.68,0.51)$ & $-0.08(-0.68,0.58)$ \\
\hline Traumatic stress: Hyper-arousal & $-0.14(-0.53,0.66)$ & $-0.01(-0.63,0.63)$ \\
\hline Traumatic stress & $0.11(-0.68,0.51)$ & $-0.03(-0.65,0.61)$ \\
\hline Loneliness & $-0.07(-0.64,0.56)$ & $-0.04(-0.66,0.60)$ \\
\hline Mindfulness & $-0.10(-0.66,0.53)$ & $0.01(-0.62,0.63)$ \\
\hline Gratitude & $-0.03(-0.62,0.58)$ & $0.21(-0.49,0.74)$ \\
\hline Vitality/fatigue & $0.39(-0.28,0.80)$ & $0.55(-0.12,0.88)$ \\
\hline Bodily Pain & $0.14\left(\begin{array}{lll}-0.50 & 0.69)\end{array}\right.$ & $-0.38(-0.81,0.33)$ \\
\hline Physical well-being & $0.22(-0.44,0.72)$ & $-0.39(-0.82,0.31)$ \\
\hline Mental well-being & $0.07(-0.56,0.64)$ & $0.13(-0.54,0.70)$ \\
\hline \multicolumn{3}{|l|}{ Biomarkers } \\
\hline Salivary cortisol (AM) & $-0.08(-0.65,0.55)$ & $0.21(-0.45,0.72)$ \\
\hline Salivary cortisol (PM) & $0.25(-0.41,0.74)$ & $0.37(-0.30,0.79)$ \\
\hline Change in salivary cortisol/hr ${ }^{\mathrm{a}}$ & $0.41(-0.27,0.81)$ & $0.04(-0.57,0.62)$ \\
\hline
\end{tabular}

CI Confidence Interval, FCR Fear of Cancer Recurrence 